\title{
Toxicité et mode d'action des extraits de Carica papaya L. (Caricaceae) sur Macrotermes bellicosus Rambur (Isoptera ; Macrotermitinae) *
}

\author{
Annick Tahiri ${ }^{1}$ \\ Mathias Assi ${ }^{2}$ \\ Augustin Amissa ${ }^{3}$ \\ ${ }^{1}$ Université de Cocody \\ UFR biosciences \\ Labo de biologie et d'endocrinologie \\ 22 BP 582 \\ Abidjan \\ Côte d'Ivoire \\ <tayaman2@yahoo.fr> \\ 2 UFR Biosciences \\ Labo de chimie \\ Université de Cocody \\ 22 BP 582 \\ Abidjan \\ Côte d'Ivoire \\ <askomath@yahoo.fr> \\ 3 INP-HB \\ Laboratoire de chimie \\ Yamoussokro \\ Côte d'Ivoire \\ <aamissa@yahoo.fr>
}

\begin{abstract}
Résumé
Les propriétés pesticides des extraits totaux de différents organes du papayer Carica papaya L. (Caricaceae) ont été testées sur l'espèce de termite ravageur Macrotermes bellicosus. L'effet de l'extrait alcoolique des graines contenues dans le fruit vert et celui des extraits hexaniques de la pulpe et des graines sont les plus toxiques. Ces trois extraits possèdent des doses léthales (DL50) similaires et les plus faibles (respectivement $0,15 \pm 0,0 \mathrm{mg} / \mathrm{L}, 0,16 \pm 0,0 \mathrm{mg} / \mathrm{L}$ et $1,06 \pm 0,0 \mathrm{mg} / \mathrm{L}$ ). Ils peuvent justifier de façon satisfaisante l'usage en milieu traditionnel du papayer comme insecticide. L'extrait alcoolique de graines et l'extrait hexanique de la pulpe de papaye, les plus toxiques, ont leurs actions optimales à faible dose. Les autres extraits ont leur toxicité qui croît aux doses supérieures. Comme mode d'action, le contact et l'inhalation sont les facteurs essentiels à l'efficacité des extraits. Ils sont de plus capables de se transmettre dans la colonie lors des tâches sociales. Mais ils n'agissent pas par ingestion et ils sont inhibiteurs de la prise alimentaire vis-à-vis du termite.
\end{abstract}

Mots clés : Carica papaya; Côte d'Ivoire ; plante pesticide ; termite.

Thèmes : pathologie ; productions végétales ; ressources naturelles et environnement.

\section{Abstract \\ Toxicity and mode of action of Carica Papaya L. (Caricaceae) extracts on Macrotermes bellicosus Rambur (Isoptera ; Macrotermitinae)}

The pesticide characteristics of the total extracts of different organs of the papaya tree Carica papaya L. (Caricaceae) were tested on the Macrotermes bellicosus termite species. The effect of these extracts, especially the alcoholic extract contained in the grains of the immature fruit and the hexane extracts contained in the pulp and the grains, are the most toxic. The three extracts have similar and the lowest LD50 (respectively 0,15 $\pm 0,0 \mathrm{mg} / \mathrm{L}, 0,16 \pm 0,0 \mathrm{mg} / \mathrm{L}$ and $1,06 \pm 0,0 \mathrm{mg} / \mathrm{L}$ ). The use of the papaya tree in a traditional environment as a pesticide is greatly justified. The effectiveness of the extracts increases with the concentration of the extract, except in the most toxic grain alcohol extract and the pulp hexane extract, which are more effective in low concentrations. Concerning the mode of action of the papaya tree, contact and inhalation are the main factors of their effectiveness. They can also be transmitted in the colony during social tasks. They do not, however, act when ingested and they are appetite-inhibiting for termites.

Key words: Carica papaya; Ivory Coast; pesticidal plants; termite.

Subjects: natural resources and environment; pathology; vegetal productions. 
ar les attaques et les dégâts qu'ils occasionnent, les termites constituent un des plus grands fléaux de l'agroforesterie tropicale et une contrainte majeure pour les agriculteurs. Dans la lutte contre ces insectes, les intrants chimiques, bien que coûteux et complexes, sont les plus employés par les agriculteurs (Cowie et Wood, 1989). Malgré l'utilisation de ces pesticides, les attaques des termites persistent (Logan et al., 1990).

Avec l'intensification de l'agriculture et les restrictions dans l'usage des pesticides pour leurs effets toxiques sur l'environnement, nous avons voulu valoriser des connaissances locales en matière de plantes à propriétés pesticides. Nous avons ainsi axé nos travaux vers la recherche de nouveaux biopesticides. Traditionnellement, les paysans utilisent des plantes pour lutter contre les termites nuisibles. Le papayer est décrit comme toxique sur les termites par Yaga (1973). Cet auteur montre en laboratoire que l'extrait aqueux du fruit vert du papayer est toxique sur les ouvriers adultes du termite Coptotermes formosanus. Cette plante est également décrite comme toxique sur d'autres insectes que le termite. L'extrait aqueux de feuilles du papayer, mélangé à l'eau d'irrigation, limite les ravages des insectes phytophages tels que les chenilles et les pucerons, dans les cultures marâichères en Côte d'Ivoire (non publié ${ }^{1}$ ). Au Bénin, les extraits aqueux du fruit vert du papayer sont utilisés traditionnellement dans la lutte contre tous les stades de la bruche Callosobruchus maculatus F., nuisible aux fleurs et aux gousses du niébé (non publié2). Aussi, nous avons vérifié le fondement de l'utilisation traditionnelle de cette plante dans les campagnes en lutte antitermites et nous avons plus particulièrement recherché le mode d'action de cette plante sur les termites.

Le choix de cette étude part des constats suivants :

- malgré les nombreux traitements chimiques utilisés en Côte d'Ivoire, les cultivateurs restent impuissants face aux ravages et aux pertes occasionnées par les termites $(96 \%$ des dégâts sur des parcelles expérimentales d'hévéa et $85 \%$ des ravages sur des parcelles expérimentales de maïs sont liés aux termites) (Tahiri, 2010) ;

\footnotetext{
1 Travaux de Bekon K.A., 1998.

2 Travaux de Ahohuendo et al., 2005.
}

- les méthodes traditionnelles à base de plantes pour la lutte contre les insectes ravageurs des cultures sont de plus en plus délaissées aux profits des intrants chimiques, délicats à utiliser, potentiellement toxiques contre les humains et onéreux pour le paysan dont les conditions de vie se dégradent.

L'objectif général de la présente étude est de proposer une solution de lutte autre que l'utilisation des intrants chimiques, efficace, plus accessible aux paysans, sans menace pour l'environnement et pour l'homme et, moins coûteuse.

Plus spécifiquement, il s'agit :

- d'étudier la toxicité directe des différents extraits totaux aqueux, alcoolique et hexanique des différents organes du papayer sur les ouvriers adultes d'une espèce de termite ravageur, Macrotermes bellicosus Rambur ;

- de déterminer: i) si la mortalité du termite peut résulter ou non d'une consommation de l'extrait ; ii) si l'extrait est toxique sans être en contact avec le termite (test d'inhalation); iii) si les ouvriers qui ont été en contact avec l'extrait peuvent contaminer des ouvriers qui n'ont pas été en contact avec le produit (test de transmission); et iv) si les ouvriers, lorsqu'ils ont le choix, sont capables de détecter le produit et de l'éviter (test de choix) ;

- d'évaluer la dose létale $\left(\mathrm{DL} 50^{3}\right)$ des extraits en 24 heures ;

- de déterminer la persistance d'efficacité des extraits actifs du papayer.

\section{Matériel et méthode}

\section{Site d'étude}

Les travaux se sont déroulés à l'université de Cocody située à Abidjan, en Côte d'Ivoire.

\section{Matériel végétal}

Notre choix s'est porté sur le papayer Carica papaya L. (Caricaceae), très courant en Côte d'Ivoire. La composition chimique des feuilles du papayer et de son fruit est connue (Busson, 1968; Fortin et al., 2000). En revanche, peu de travaux mentionnent ses propriétés pesticides.

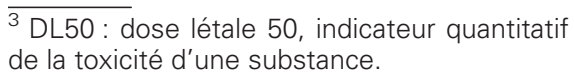

Les fruits et les feuilles sont utilisés comme insecticides contre les termites (Yaga, 1973), contre les chenilles, les pucerons et les bruches (non publié ${ }^{4}$ ).

\section{Matériel animal}

Macrotermes bellicosus Rambur (sousfamille des Macrotermitinae) a été choisie pour les tests biologiques en raison de son impact sur de nombreuses cultures, de son abondance dans la zone d'étude et de son nid épigé permettant la capture en grand nombre des ouvriers. Les ouvriers étudiés dans ces travaux ne proviennent pas d'élevage, mais d'une même colonie issue du même nid du campus de Cocody. Chez cette espèce champignonniste, les ouvriers sont de deux tailles grands et petits. Le dimorphisme entre la taille de la tête des petits et des grands ouvriers permet de les séparer pour nos expériences.

\section{Préparation des extraits totaux}

La plante a été récoltée de mai à septembre 2005 et de mai à septembre 2006. Les résultats concernent ces deux séries de prélèvements, sans distinguer les années. Les extraits totaux aqueux, alcoolique et hexanique de feuilles, de graines et de pulpe du fruit sont réalisés selon la méthode d'extraction successive classique par des solvants de différentes polarités (Kaushik et Vir, 2000). L'organe végétal est d'abord épuisé par l'hexane, ensuite par le méthanol, et enfin, par l'eau. Les solutions recueillies sont évaporées par évaporation rotative au rotavapor, pour obtenir des extraits totaux hexanique (huileux), méthanolique et aqueux, qui sont séchés sous vide.

\section{Calcul du taux (en \%) du rendement à l'extraction}

Le rendement à l'extraction de chaque fraction (R) se définit comme le rapport de la quantité de l'extrait (E) sur la quantité de matière sèche du produit (MS) :

$$
\mathrm{R}=\mathrm{E} / \mathrm{MS} \times 100
$$

\footnotetext{
$\overline{4 \text { Travaux }}$ de Bekon et al., 1998 et de Ahohuendo et al., 2005.
} 


\section{Préparation des formulations}

Une solution-mère à 10 \% est préparée à partir de chaque extrait séché avec le solvant correspondant avant d'être testée sur 1 gramme de poids frais des petits ouvriers (PO) adultes du termite $M$. bellicosus (soit sur 136 petits ouvriers), aux doses de 10, 20, 50 et $100 \mu \mathrm{L}$ par boîte, soit aux quatre teneurs suivantes : 1, 2, 5 et $10 \mathrm{mg}$ d'extrait/L. Le témoin est traité aux mêmes doses du solvant correspondant.

\section{Tests biologiques}

Cinq tests biologiques sont réalisés selon les protocoles de Delgarde et RoulandLefèvre (2002) :

1. Le test de toxicité directe permet de mesurer les réponses des termites à un sol traité avec l'insecticide ;

2. Le test de toxicité par consommation permet de déterminer si la mortalité des petits ouvriers résulte ou non d'une consommation de l'insecticide et précise l'importance de la consommation du produit dans cette mortalité ;

3. Le test de toxicité par inhalation sert à étudier si les émanations de l'insecticide sont toxiques ;

4. Le test de toxicité par transmission est utilisé pour déterminer si des grands ouvriers qui ont été en contact avec l'insecticide pendant 1 heure et demie, puis transférés avec des petits ouvriers qui n'ont pas été en contact avec le produit, peuvent contaminer les petits ouvriers après 2 heures de présence des deux catégories d'ouvriers ;

5. Le test d'évitement de l'extrait permet de déterminer si les ouvriers, lorsqu'ils ont le choix, sont capables de détecter le produit et de l'éviter.

Les tests sont réalisés à la température ambiante du laboratoire comprise entre 27 et $28{ }^{\circ} \mathrm{C}$. Les tests de toxicité directe, par consommation et par transmission, sont réalisés dans une petite boîte rectangulaire en plexiglass de 95 × 65 × $20 \mathrm{~mm}$ de hauteur contenant 7 grammes de terre humidifiée avec $2 \mathrm{ml}$ d'eau distillée. Les tests d'inhalation et par évitement sont réalisés dans une grande boîte en plexiglass de 180 x 120 x 70 mm de hauteur contenant 17 grammes de terre tamisée et humidifiée avec $5 \mathrm{ml}$ d'eau distillée. À l'aide d'une micropipette, les doses sont déposées soit sur la terre (pour les tests de toxicité directe et par transmission), soit sur des morceaux de papier Whatman
$\mathrm{N}^{\circ} 1$ de $4 \mathrm{~cm}^{2}$ (pour les tests de consommation, d'inhalation et d'évitement). Après dépôt, les boîtes sont séchées à l'air libre durant 1 heure. Les petits ouvriers de $M$. bellicosus sont ensuite introduits dans ces dispositifs qui sont fermés et ne permettent pas de circulation d'air. Chaque solution d'extrait est testée aux quatre doses citées. Chaque dose est reprise dix fois pour l'ensemble des tests. Chaque boîte témoin est traitée avec le solvant correspondant.

La mortalité des petits ouvriers a été déterminée 24 heures après les traitements. La DL50 est calculée. La surface de chaque papier Whatman recouverte de placage de terre et celle consommée $\left(\mathrm{mm}^{2} /\right.$ ouvrier) sont mesurées chaque jour avec un micromètre oculaire adapté à une loupe. La quantité d'extrait ingérée par ouvrier par jour (en ppm) est calculée.

\section{Persistance d'efficacité (en jours) de l'extrait le plus actif}

Dans une petite boite en plexiglass de $95 \times 65 \times 20 \mathrm{~mm}$ de hauteur contenant 7 grammes de terre humidifiée avec $2 \mathrm{ml}$ d'eau distillée, la terre est traitée à la dose la plus efficace de l'extrait. Les petits ouvriers sont retirés et remplacés toutes les 24 heures par de nouveaux pendant 7 jours. Les boites témoins sont traitées avec le solvant correspondant. Les petits ouvriers morts sont comptés jusqu'à ce que la quantité dans les boîtes essais et dans les témoins ne soit pas significativement différente. Le pourcentage de mortalité est calculé.

\section{Calcul du pourcentage de mortalité}

Le pourcentage de mortalité (Pc) est calculé selon le rapport du nombre d'individus morts observé sur le nombre total de termites :

\section{Tableau 1. Rendement d'extractions successives des différentes parties de Carica papaya.}

Table 1. Successive extraction output of the different parts of Carica papaya.

\begin{tabular}{lccc}
\hline & Extrait hexanique (\%) & Extrait alcoolique (\%) & Extrait aqueux (\%) \\
\hline Feuille & 6 & 13 & 11 \\
Graine & 15 & 18 & 17 \\
Pulpe & 12 & 15 & 13 \\
\hline
\end{tabular}

Pc $=$ Mortalités observés/nombre total termites x 100

\section{Analyses statistiques}

Les données recueillies lors des tests biologiques sont traitées au moyen du logiciel Statistica (2001).

Le diagramme en boîte, l'estimation boostrap, les tests non paramétriques de Newman-Keuls et de Kruskal-Wallis (au seuil de $5 \%$ ) et les tests de corrélation ont été utilisés. La DL50 est calculée par l'analyse Probit sur la base des mortalités obtenues après 24 heures sur différentes doses.

\section{Rendement d'extractions successives des différentes parties de $C$. papaya}

Les quantités successives extraites par chacun des trois solvants exprimées en pourcentage de la quantité totale extraite sont indiquées dans le tableau 1.

\section{Toxicité directe des extraits de $C$. papaya}

Sept groupes d'extraits classés des plus efficaces au moins actifs selon leur toxicité (DL50), sur le termite $M$. bellicosus, se distinguent (tableau 2). Dans le groupe 1, et en tête de liste, se retrouvent les extraits pulpe et de graines. Ces extraits possèdent une toxicité supérieure à l'ensemble des autres extraits de la plante et des DL50 similaires. Dans le groupe 2 , se retrouve l'extrait aqueux de feuilles. En revanche, l'extrait alcoolique de la pulpe est le moins toxique (tableau 2). Les pourcentages de mortalité des petits ouvriers obtenus après traitement avec cet extrait alcooliques de graines et hexaniques de 
restent en deçà de $50 \%$ de la population. Les extraits les plus toxiques (alcoolique de graines et hexanique de pulpe) ont leur action optimale à faible dose (1 mg/L). À dose élevée (10 mg/L), ils sont moins performants. Les toxicités des autres extraits sont corrélées positivement avec les doses.

\section{Persistance d'efficacité (en jours) des différents extraits de $C$. papaya}

Les extraits de graines de C. papaya possèdent les meilleures persistances. Entre 3,7 jours et 4,3 jours, les extraits restent actifs (les taux de mortalité des petits ouvriers sont significativement supérieurs à celui obtenu chez le témoin au seuil de $5 \%)($ tableau 3)

\section{Mode d'action de l'extrait alcoolique de graines de $C$. papaya le plus toxique sur le termite}

\section{Toxicité par consommation}

Les papiers témoins et les papiers traités à l'extrait sont visités par le termite comme le montrent les placages de terre. Mais le termite ne consomme pas les papiers traités.

En revanche, la surface moyenne de papier consommée chez le témoin est de $5 \mathrm{~mm}^{2}$, soit $0,04 \mathrm{~mm}^{2}$ /ouvrier (tableau 4 ). La quantité d'extrait ingérée par les ouvriers en fin d'expérience est nulle. Or, les pourcentages de mortalité obtenus chez les ouvriers traités sont significativement supérieurs à celui du témoin $(\mathrm{H}=9,33 ; p=0,000 ; \mathrm{N}=50)$. Il n'y a donc pas de corrélation entre la mortalité des ouvriers et la consommation de l'extrait pour les doses testées à 24 heures ( $\mathrm{R}=0,45 ; \quad \mathrm{N}=50 ; p=0,220)$. L'effet toxique de l'extrait alcoolique de graines de C. papaya n'est donc pas lié à son ingestion par le termite.

Nous allons préciser par les tests suivants les autres voies d'actions du produit.

\section{Toxicité par inhalation}

L'extrait n'agissant pas par ingestion, il est intéressant de voir s'il peut être toxique sans être en contact avec le termite. Aux doses de 1, 2, 5 et $10 \mathrm{mg} / \mathrm{L}$, l'inhalation de l'extrait entraîne un taux de mortalité significativement plus élevé que celui obtenu chez le témoin à 24 heures et à 48 heures après le traitement $(\mathrm{H}=9,47$;

Tableau 2. Classement par ordre décroissant des DL50 des extraits de Carica papaya sur les petits ouvriers de Macrotermes bellicosus dans le test de toxicité directe.

Table 2. LD50 classification in descending order of Carica papaya extract on small Macrotermes billicosus workers in the direct toxicity test.

\begin{tabular}{llr}
\hline Ordre d'efficacité & \multicolumn{1}{c}{ Extrait } & DL50 en $\mathbf{2 4} \mathbf{~ h ~}$ (mg/L) \\
\hline 1 & Alcoolique graine & $0,15 \pm 0 \mathrm{a}$ \\
2 & Hexanique pulpe & $0,16 \pm 0 \mathrm{a}$ \\
3 & Hexanique graine & $0,6 \pm 0 \mathrm{ab}$ \\
4 & Aqueux Feuille & $2 \pm 0 \mathrm{~b}$ \\
5 & Alcoolique feuille & $10,3 \pm 0 \mathrm{c}$ \\
6 & Hexanique feuille & $18 \pm 0 \mathrm{~d}$ \\
7 & Aqueux graine & $23,5 \pm 0 \mathrm{e}$ \\
8 & Aqueux pulpe & $146 \pm 0 \mathrm{f}$ \\
9 & Alcoolique pulpe & $152,3 \pm 0,2 \mathrm{~g}$ \\
\hline
\end{tabular}

À l'intérieur d'une même colonne, les valeurs suivies des mêmes lettres ne sont pas significativement différentes au seuil de $5 \%$ selon le test de Kruskal-Wallis ( $p<0,05$; Anova - analysis of variance). DL50 : dose létale 50, indicateur quantitatif de la toxicité d'une substance.

\section{Tableau 3. Persistance d'efficacité* (en jours) des différents extraits de Carica papaya.}

Table 3. Persistence of efficiency* (in days) of the different Carica papaya extracts.

\begin{tabular}{lccc}
\hline & Extrait hexanique & Extrait alcoolique & Extrait aqueux \\
\hline Feuille & $3,3 \pm 0,4 \mathrm{~b}$ & $3,7 \pm 0,4 \mathrm{a}$ & $3,3 \pm 0,4 \mathrm{~b}$ \\
Graine & $3,7 \pm 0,4 \mathrm{a}$ & $3,7 \pm 0,4 \mathrm{a}$ & $4,3 \pm 0,4 \mathrm{a}$ \\
Pulpe & $3,3 \pm 0,4 \mathrm{~b}$ & $3,2 \pm 0,4 \mathrm{~b}$ & $3,7 \pm 0,4 \mathrm{a}$
\end{tabular}

* La persistance d'efficacité est la durée de l'activité résiduelle d'un extrait qui engendre des taux de mortalité supérieurs au témoin au seuil de $5 \%$.

Moyenne de 10 répétitions + écart type $(N=90)$. À l'intérieur d'une même colonne, les valeurs suivies des mêmes lettres ne sont pas significativement différentes au seuil de $5 \%$ selon le test de KruskalWallis ( $p<0,05 ;$ Anova - analysis of variance).

Extrait hexanique $(H=5,787 ; p=0,022 ; N=90)$; extrait alcoolique $(H=5,829 ; p=0,025 ; N=90)$; extrait aqueux $(H=6,682 ; p=0,024 ; N=90)$.

$p=0,000 ; \mathrm{N}=50)$. En revanche, les taux de mortalité ne sont pas significativement différents entre les doses $(\mathrm{H}=9,187$; $p=0,081 ; \mathrm{N}=40)$.

\section{Toxicité par transmission}

Aux doses de 1, 2 et $5 \mathrm{mg} / \mathrm{L}$, le taux de mortalité des petits ouvriers de $M$. bellicosus mis en contact avec les grands ouvriers traités avec l'extrait est significativement plus élevé que celui obtenu chez le témoin $(\mathrm{H}=19,66 ; p=0,000 ; \mathrm{N}=50)$ (figure 1). Le TL $50^{5}$ moyen de $0,9 \pm 0,3$ jour est significativement inférieur au TL50 de 1,8 $\pm 0,1$ jours obtenu chez le témoin $(\mathrm{H}=19,48 ; p=0,000 ; \mathrm{N}=50)$. Cet extrait est donc favorable à la transmission dans les colonies.

\footnotetext{
5 TL50 : temps létal 50, (temps moyens pour lesquels on observe $50 \%$ de morts).
}

\section{Évitement de l'extrait}

Lorsque le choix se présente, les papiers témoins et les papiers traités à l'extrait sont visités par le termite comme le montrent les placages de terre. Mais aucun papier, traité ou témoin disposé à proximité, n'est consommé par les ouvriers de $M$. bellicosus (tableau 5). L'inhibition de la prise alimentaire par l'extrait s'exerce aussi bien sur la consommation du papier traité que sur celle du papier non traité disposé à proximité.

\section{Discussion}

Toutes les parties du papayer (feuilles, pulpe et graines du fruit) s'avèrent plus ou moins toxiques pour $M$. bellicosus. 


\section{Tableau 4. Effet de l'extrait alcoolique de graines de Carica papaya sur l'activité de récolte des ouvriers de Macrotermes bellicosus (test par consommation).}

Table 4. Effect of the Carica papaya grain alcohol extract on the Macrotermes billicosus workers' collection activity (consumption test).

\begin{tabular}{cccc}
\hline $\begin{array}{c}\text { Dose d'extrait alcoolique } \\
\text { de graine de } \mathbf{C} \text { papaya } \\
\text { (mg/L) }\end{array}$ & $\begin{array}{c}\text { Surface placage cumulée } \\
\text { (mm²/ov) }\end{array}$ & $\begin{array}{c}\text { Surface consommée } \\
\text { cumulée de papier } \\
\text { (mm } \mathbf{m} / \mathbf{o v})\end{array}$ & $\begin{array}{c}\text { Quantité d'extrait } \\
\text { ingérée cumulée } \\
\text { (en } \mathbf{p p m} / \mathbf{o v})\end{array}$ \\
\hline 0 & $0,30 \pm 0,23 \mathrm{a}$ & $0,04 \pm 0 \mathrm{~b}$ & $0 \mathrm{a}$ \\
1 & $4,50 \pm 5,46 \mathrm{ab}$ & $0 \mathrm{a}$ & $0 \mathrm{a}$ \\
2 & $2,18 \pm 1 \mathrm{~b}$ & $0 \mathrm{a}$ & $0 \mathrm{a}$ \\
5 & $2,23 \pm 1,31 \mathrm{~b}$ & $0 \mathrm{a}$ & $0 \mathrm{a}$ \\
10 & $3 \pm 5 \mathrm{~b}$ & $0 \mathrm{a}$ & $0 \mathrm{a}$
\end{tabular}

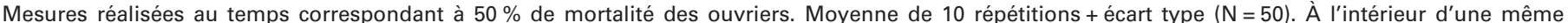

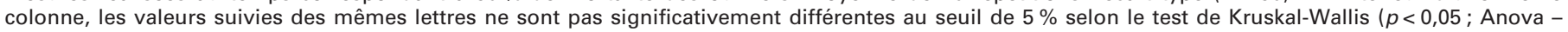
analysis of variance).

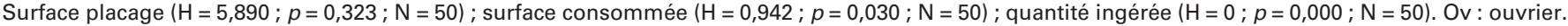

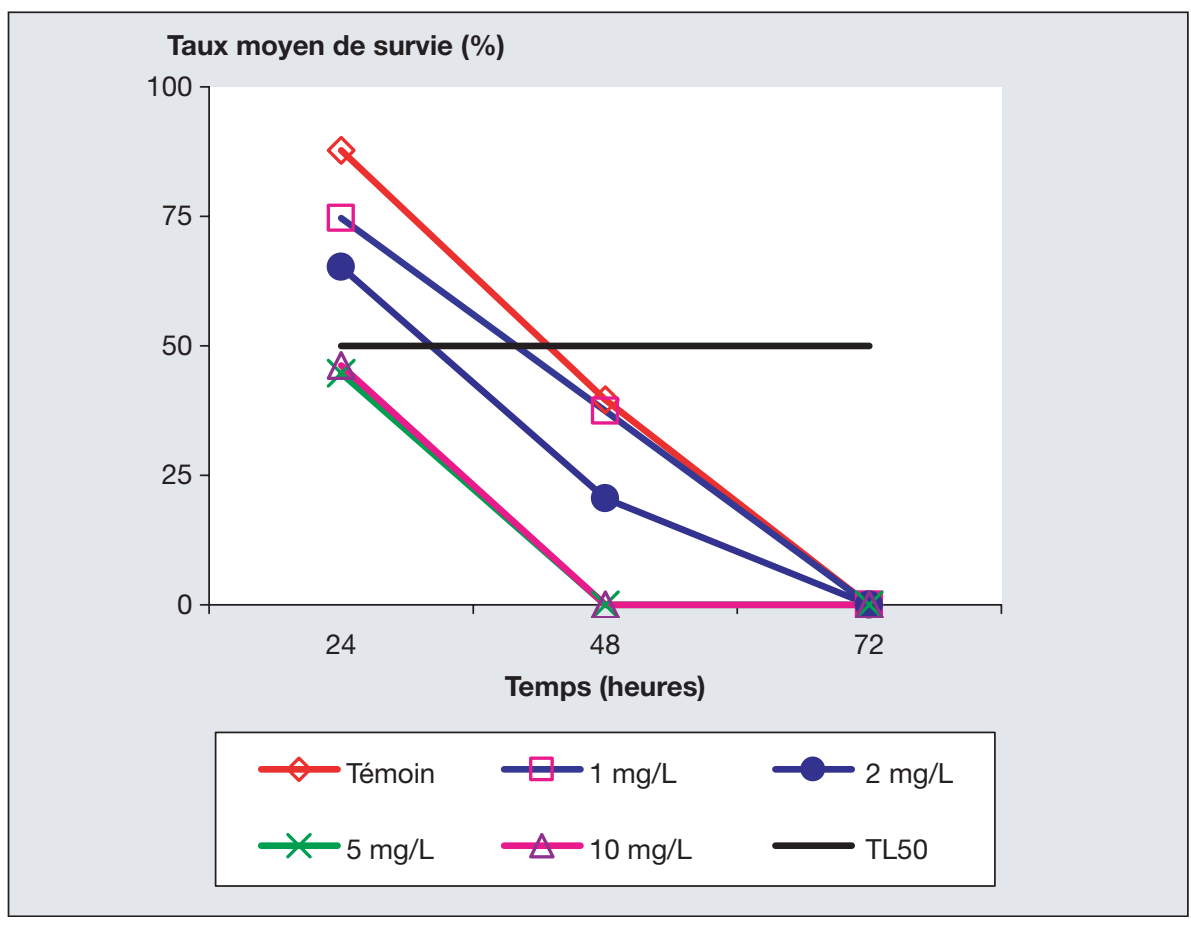

Figure 1. Taux de survie des petits ouvriers de Macrotermes bellicosus au contact des grands ouvriers traités I'extrait alcoolique de graines de Carica papaya.

Figure 1. Survival rate of small Macrotermes bellicosus workers in contact with large workers treated with the Carica papaya grain alcohol extract.

Les extraits du fruit vert (extrait alcoolique de graines et extraits hexaniques de pulpe et de graines) sont les plus toxiques. Les constituants du papayer contribuent à expliquer l'action insecticide potentielle. L'extrait alcoolique de graines du papayer contient des terpénoïdes, des tanins et des alcaloïdes (Tahiri, 2010). Les effets insecticides de ces constituants ont été mentionnés par plusieurs auteurs. Les terpénoïdes ont des propriétés insec- ticide, fongicide, répulsive et antiappétante (Wardell, 1987; Fortin et al., 2000). Les tanins possèdent des propriétés insecticides, larvicides et répulsifs (Zhang et al., 1990). Les alcaloïdes induisent des effets toxiques vis-à-vis des insectes (Appert et Deuse, 1982; Bouchelta et al., 2005). Les deux extraits les plus performants, aux doses élevées, présentent un rapport dose-effet non linéaire. Nos observations sont similaires à celles des travaux de Fournier (1998), de Delgarde et Rouland-Lefèvre (2002) et de Reinhard et al. (2002), avec l'utilisation de signaux chimiques comme les phéromones et l'attractant X94 (thiophane de méthyle) sur les termites. Ces auteurs l'expliquent par des interactions chimiques des composants pouvant entraîner la saturation des processus tels que leur absorption ou leur activation métabolique.

Les extraits de graines de papayer possèdent les meilleures persistances d'efficacité et de bons rendements d'extraction.

Nos résultats montrent également que le contact et l'inhalation sont les deux facteurs essentiels à l'efficacité des extraits du papayer sur le termite. Les substances toxiques peuvent donc pénétrer à travers la cuticule et les stigmates de l'insecte. Ils n'agissent pas par ingestion et ils sont inhibiteurs de la prise alimentaire vis-à-vis du termite. Leur utilisation en appât contre cet insecte peut donc poser des problèmes. Ils sont en revanche capables de se transmettre à partir d'individus traités dans la colonie, lors des tâches sociales, par contact et par léchage, ce qui est une qualité recherchée dans un termiticide.

Les extraits n'ont plus d'effet 72 à 96 heures après le traitement. À l'instar de nombreux autres insectes phytophages, le termite doit pouvoir détoxifier les composés toxiques en produisant des enzymes qui sont impliqués dans les mécanismes métaboliques de la détoxication de substances organiques polluantes (Scott, 1999).

Des techniques simples de gestion des attaques des termites en milieu de culture par des extraits aqueux de graines de papayer peuvent être facilement réalisées par les cultivateurs. 


\section{Tableau 5. Effet de l'extrait alcoolique de graines de Carica papaya sur l'activité de récolte des ouvriers de Macrotermes bellicosus (test par évitement).}

Table 5. Effect of the Carica papaya grain alcohol extract on the Macrotermes billicosus workers' collection activity (avoidance test).

\begin{tabular}{ccc}
\hline $\begin{array}{c}\text { Dose d'extrait alcoolique } \\
\text { de graine de } \boldsymbol{C} \text {. papaya } \\
\text { (mg/L) }\end{array}$ & $\begin{array}{c}\text { Surface placage } \\
\text { cumulée } \\
\left(\mathbf{m m}^{\mathbf{2}} / \mathbf{o v}\right)\end{array}$ & $\begin{array}{c}\text { Surface de papier } \\
\text { consommée cumulée } \\
\left(\mathbf{m m}^{\mathbf{2}} / \mathbf{o v}\right)\end{array}$ \\
\hline Témoin & $3 \pm 0,67 \mathrm{a}$ & $0 \mathrm{a}$ \\
$1 \mathrm{mg} / \mathrm{L}$ & $1,64 \pm 0,50 \mathrm{a}$ & $0 \mathrm{a}$ \\
Témoin & $3 \pm 2,87 \mathrm{a}$ & $0 \mathrm{a}$ \\
$2 \mathrm{mg} / \mathrm{L}$ & $1,80 \pm 1,48 \mathrm{a}$ & $0 \mathrm{a}$ \\
Témoin & $3,25 \pm 5,26 \mathrm{a}$ & $0 \mathrm{a}$ \\
$5 \mathrm{mg} / \mathrm{L}$ & $2,43 \pm 2,60 \mathrm{a}$ & $0 \mathrm{a}$ \\
Témoin & $3 \pm 2,15 \mathrm{a}$ & $0 \mathrm{a}$ \\
$10 \mathrm{mg} / \mathrm{L}$ & $1,41 \pm 1,60 \mathrm{a}$ & $0 \mathrm{a}$ \\
\hline
\end{tabular}

Mesures réalisées au temps correspondant à $50 \%$ de mortalités des ouvriers. Moyenne de 10 répétitions + écart type $(\mathrm{N}=80)$.

À l'intérieur d'une même colonne, les valeurs suivies des mêmes lettres ne sont pas significativement différentes au seuil de $5 \%$ selon le test de Kruskal-Wallis ( $p<0,05$; Anova - analysis of variance).

Surface placage $(H=9,718 ; p=0,082 ; N=80)$; surface consommée $(H=0 ; p=0,000 ; N=80)$. Ov : ouvrier.

Les doses à appliquer sont réalisables (en laboratoire ; la dose expérimentale de $1,6 \mathrm{~kg}$ d'extrait/ha a donné des résultats satisfaisants).

\section{Conclusion}

Bien que moins actifs que les insecticides vendus sur le commerce comme antitermites, les extraits naturels du papayer peuvent être une autre solution à la place des pesticides dans la lutte contre les termites. Des compléments d'analyses sur l'identification des composés actifs sur le termite, sur les capacités de formulations et sur les facteurs qui peuvent compromettre leurs efficacités sur le terrain sont à évaluer en milieu naturel.

\section{Références}

Appert J, Deuse J. Les ravageurs des cultures vivrières et maraîchères sous les tropiques. Paris: Maisonneuve \& Larose, 1982.

Bouchelta A, Boughdad A, Blenzar A. Effets biocides des alcaloïdes, des saponines et des flavonoïdes extraits de Capsicum frutescens L. (Solanaceae) sur Bemisia tabaci (Gennadius) (Homoptera: Aleyrodidae). Biotechnol Agron Soc Environ 2005 ; 9 : 21-30.
Busson HM. Plantes alimentaires de l'Ouest africain. Marseille: Leconte ed, 1968.

Cowie RH, Wood TG. Damage to crops, forestry and rangeland by fungus-growing termites (Termitidae: Macrotermitinae) in Ethiopia. Sociobio $\log y 1989 ; 15: 139-53$

Delgarde S, Rouland-Lefèvre C. Efficacy of Actara 25 WG on three species of Brazilian termite (Isoptera: nasutitermitinae). Sociobiology $2002 ; 40: 669-710$

Fortin D, Lô M, Maynart G. Plantes médicinales du Sahel. Dakar (Sénégal) : Enda éditions, 2000.

Fournier J. Chimie des pesticides. Cultures et Techniques - ACCT. Vienne: Les Trois Moutiers, 1998.

Kaushik N, Vir S. Variations in fatty acid and composition of neem seeds collected from the Rajasthan State of India. Biochem Soc Trans $2000 ; 28: 880-2$.

Logan JWM, Cowie RH, Wood TJ. Termite (Isoptera) control in agriculture band forestry non chemical methods, a review. Bull Entomol Res $1990 ; 80: 309-30$.

Reinhard J, Lacey M, Ibarra F, et al. Hydroquinone. A general phagostimulating pheromone in termites. J Chem Ecol $2002 ; 28: 1-14$.

Scott JC. Cytochromes and insecticide resistance. Insect Biochem Mol Biol 1999; 29: 757-77

Tahiri A. Termites ravageurs de Hevea brasiliensis (Kunth) Müll. Arg., 1775 dans les départements de Daoukro et d'Agboville (Côte d'Ivoire) : lutte par utilisation d'extraits de 6 plantes locales contre Macrotermes bellicosus Rambur 1842 Thèse d'État ès-sciences naturelles, université de Cocody, Abidjan 2010.

Wardell DA. Control of termites in nurseries and young plantations in Africa: established practices and alternative courses of action. Commonw Forestr Rev 1987 ; 66 : 77-89.

Yaga S. Termiticidal activity of immature fruit juice from papaya. Mokuzai Gakkaishi 1973; 19 : 349-50.

Zhang J, Takahashi K, Kono Y, et al. Bioactive condensed tannins from bark: chemical properties, enzyme inhibition and anti-plant-viral activities. J pesticide Sci $1990 ; 15: 585-91$. 